\title{
Depth dependence of the effective properties of subwavelength gratings
}

\author{
Philippe Lalanne \\ Institut d'Optique Théorique et Appliquée, Centre National de la Recherche Scientifique, BP 147, \\ F91403 Orsay Cedex, France \\ Dominique Lemercier-Lalanne \\ Lycée Janson de Sailly, F75015 Paris, France
}

Received May 1, 1996; revised manuscript received August 29, 1996; accepted September 9, 1996

\begin{abstract}
Effective-medium theory is often helpful for understanding the behavior of subwavelength gratings with small period-to-wavelength ratios. By analytically solving Maxwell's equations in the small-depth limit, we show that the effective properties of subwavelength gratings strongly depend on the grating depth. Moreover, the effective properties are shown to depend not only on the grating structure but also on the optical indices of the surrounding media. A simple expression for the effective indices of one-dimensional (1-D) and twodimensional (2-D) gratings with arbitrary depths is proposed. Comparison with rigorous computations shows that for TE polarization of 1-D gratings the depth dependence of the effective index prediction is accurate. For TM polarization of 1-D volume gratings and for 2-D volume gratings a slight deviation between rigorous computation and the prediction is observed for approximately quarter-wave depths. For TM polarization of 1-D surface-relief gratings and for 2-D surface-relief gratings, no closed-form expression for the depth dependence of the effective indices is found, but a very sharp variation of the effective index at small thicknesses is predicted. This prediction is confirmed by rigorous computation. () 1997 Optical Society of America [S0740-3232(97)01502-0]
\end{abstract}

Key words: effective-medium theory, homogenization, subwavelength grating.

\section{INTRODUCTION}

In recent years periodic subwavelength gratings have been used to simulate homogeneous media for a variety of applications, including polarization devices, ${ }^{1}$ antireflection surfaces, ${ }^{2-5}$ narrow-band filters, ${ }^{6}$ and graded-phase diffractive elements. ${ }^{7-9}$ A periodic subwavelength grating is defined as a diffractive component that does not diffract light in the far field. Only the reflected and transmitted zero orders are propagating in the incident medium and in the substrate. As light propagates through the subwavelength grating, it experiences approximately the same behavior as if it were traveling through a homogeneous medium. The effective-medium theory (EMT) relates the parameters of a subwavelength grating to those of an effective homogeneous medium.

A detailed approach for determining the effective optical properties of media that are stratified in one direction was derived by Rytov. ${ }^{10}$ This result, which holds for lamellar gratings, was derived by matching Maxwell's boundary conditions inside the periodic structure. By using a Fourier expansion of the field inside the periodic structure, Bell et al. ${ }^{11}$ generalized Rytov's results to arbitrary one-dimensional (1-D) symmetric gratings. More recently, by using a similar approach, the present authors $^{12}$ derived the EMT of 1-D gratings with symmetric or nonsymmetric profiles. These works show that a 1-D subwavelength grating exhibits uniaxial properties in the small-period limit, i.e., when the period-to-wavelength ratio becomes infinitely small. Limited research has been done in the area of two-dimensional (2-D) grat- ings. Jackson and Coriell ${ }^{13}$ derived closed forms for the upper and lower bounds of the zero-order effective index. As was noted by Chen and Craighhead, ${ }^{9}$ these bounds are particularly helpful for designing phase plates when the index modulation is small. Motamedi et al. ${ }^{4}$ proposed an approximate solution for the zero-order effective index, inspired by the effective properties of 1-D gratings. Grann et al. ${ }^{14}$ employed rigorous coupled-wave analysis (RCWA) for numerically computing the effective index and showed that Motamedi's approximate solution is inaccurate. In Ref. 15 Bräuer and Bryngdahl used an ingenious combination of upper and lower bounds to design 2-D antireflection gratings. In Ref. 12 the present authors rigorously derived a methodology for computing the first two nonnull effective indices of 2-D symmetric gratings. No closed form was obtained, and the computation of the zero- and second-order effective index coefficients required the inversion of a matrix that has to be truncated for numerical purposes. Despite all these efforts, there are still some open questions about the behavior of subwavelength gratings and their effective properties.

In the above-mentioned works the effective indices depend only on the grating parameters, namely, the periodto-wavelength ratio and the grating relative permittivity. In this paper we mathematically show and provide numerical evidence that the effective properties of 1-D and 2 -D gratings also depend on the grating depth and on the optical indices of the surrounding homogeneous regions. In Section 2 we provide a rigorous analytical solution of grating diffraction problems in the small-depth limit, i.e., 
for grating depths much smaller than the wavelength. By comparison of the reflected and transmitted zero-order amplitudes with those provided by a homogeneous thin film with the same thickness as that of the grating, we derive closed-form expressions of the grating effective indices for the case of a normally incident plane wave. In Section 3 these closed-form expressions are simplified. By interpreting the classical EMT results of Refs. 10-12 as valid in the large-depth limit (grating depths much larger than the wavelength), we propose approximate expressions for the optical effective indices of gratings with arbitrary depths. These expressions hold for 1-D and 2 -D volume gratings and for TE polarization of 1-D surface-relief gratings. For TM polarization of 1-D surface-relief gratings and for 2-D surface-relief gratings no closed-form expression for the depth dependence of the effective indices is found, but a sharp variation of the effective index is predicted for small depths. In Section 4 the depth dependence prediction is checked with rigorous computations. Section 5 concludes with a discussion on the regions of validity of EMT. It is emphasized that EMT must be used with caution for quarter-wave thicknesses, for instance when surface-relief gratings are considered for designing single-layer antireflection coatings.

\section{EXACT SOLUTION IN THE SMALL- DEPTH LIMIT}

The 1-D grating diffraction problem considered in the following is depicted in Fig. 1. The grating region is composed of a 1-D periodic structure along the $x$ axis with an arbitrary permittivity profile $\epsilon(x)$. It is bound by two homogeneous media with refractive indices $n_{1}$ and $n_{3}$. The $z$ axis is perpendicular to the grating boundaries, and the diffraction problem is invariant in the $y$ direction. Magnetic effects are not considered in this paper, and the constant $\mu_{0}$ denotes the permeability of the periodic structure and of the surrounding regions. We denote by $c$ the speed of light in a vacuum. The grating period is denoted by $\Lambda$, and the magnitude $K$ of the grating vector is equal to $2 \pi / \Lambda$. A plane wave is normally incident from medium 1 . We denote by $\lambda$ the wavelength of the incident wave in a vacuum and denote the wave-vector magnitude by $k(k=2 \pi / \lambda)$. A temporal dependence of $\exp (j \omega t)$ of

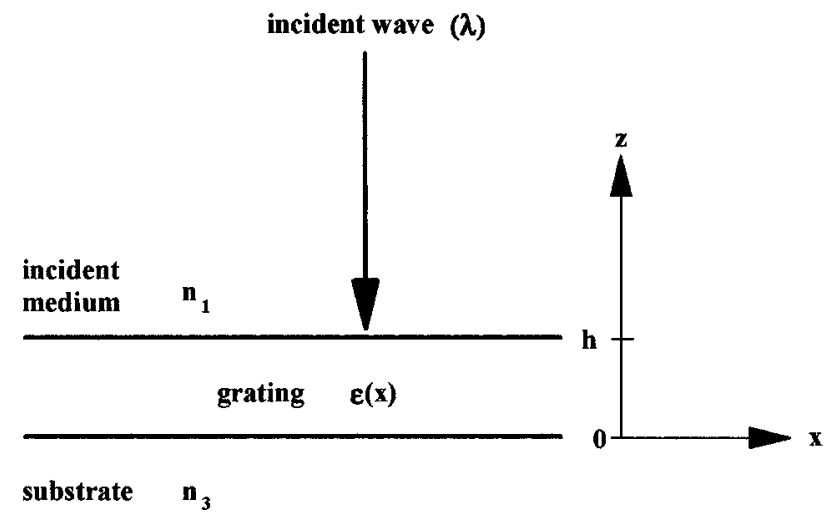

Fig. 1. General diffraction problem investigated in this paper (1-D case). the incident wave is assumed $\left(j^{2}=-1\right) . \quad \epsilon_{m}$ will denote the $m$ th Fourier coefficient of the relative permittivity.

For TE polarization (electric-field vector parallel to the grating vector) the $y$ components $E_{1, y}$ and $E_{3, y}$ of the electric fields in regions 1 and 3 are given by

$$
\begin{aligned}
E_{1, y}= & \exp \left[j n_{1} k(z-h)\right]+\sum_{i} R_{i} \\
& \times \exp \left[-j\left(n_{1}{ }^{2} k^{2}-i^{2} K^{2}\right)^{1 / 2}(z-h)\right] \exp (j i K x),
\end{aligned}
$$

$$
E_{3, y}=\sum_{i} T_{i} \exp \left[j\left(n_{3}{ }^{2} k^{2}-i^{2} K^{2}\right)^{1 / 2} z\right] \exp (j i K x),
$$

respectively. Using the Floquet theorem, we can express the $y$ component $E_{y}$ of the electric field and the $x$ component $H_{x}$ of the magnetic field in the grating region as

$$
\begin{aligned}
& E_{y}=\sum_{m} S_{m}(z) \exp (j K m x), \\
& H_{x}=j \frac{1}{\mu_{0} c} \sum_{m} U_{m}(z) \exp (j K m x) .
\end{aligned}
$$

In the following equations we denote the first derivative in the $z$ variable by a prime. By solving Maxwell's curl equations in the grating region and by identifying the $i$ th space-harmonic field components in the quasi-plane-wave basis, we obtain

$$
\begin{aligned}
& S_{i}^{\prime}=-k U_{i}, \\
& U_{i}^{\prime}=-k\left(i^{2} \alpha^{2} S_{i}-\sum_{p} \epsilon_{i-p} S_{p}\right),
\end{aligned}
$$

where $\alpha$ is a dimensionless coefficient equal to $K / k$. Except for minor notation disparities, all these equations can be found in recent papers on the coupled-wave analysis of diffraction gratings (see, for instance, Ref. 16).

We now proceed with the solution of the grating diffraction problem in the limit in which the grating depth is small compared with the wavelength $(h k \ll 1)$. We assume that the $i$ th space-harmonic field components in the grating region can be expanded into a power series in terms of $h k$. Using Eqs. (4) to express the first and second derivatives of the space-harmonic field components, we obtain the following Taylor expansions:

$$
\begin{aligned}
S_{i}(h)= & S_{i}(0)-h k U_{i}(0)+\frac{(h k)^{2}}{2}\left[i^{2} \alpha^{2} S_{i}(0)\right. \\
& \left.-\sum_{p} \epsilon_{i-p} S_{p}(0)\right]+O\left(h^{3} k^{3}\right) \\
U_{i}(h)= & U_{i}(0)-h k\left[i^{2} \alpha^{2} S_{i}(0)-\sum_{p} \epsilon_{i-p} S_{p}(0)\right] \\
& +\frac{(h k)^{2}}{2}\left[i^{2} \alpha^{2} U_{i}(0)-\sum_{p} \epsilon_{i-p} U_{p}(0)\right] \\
& +O\left(h^{3} k^{3}\right) .
\end{aligned}
$$

Equations (5) provide a linear relationship between the electromagnetic-field components at the two boundaries 
of the grating region. We also assume that the reflection and transmission coefficients, $R_{i}$ and $T_{i}$, can be expanded in a similar way:

$$
\begin{aligned}
& R_{i}=R_{i}^{(0)}+h k R_{i}^{(1)}+\frac{(h k)^{2}}{2} R_{i}^{(2)}+O\left(h^{3} k^{3}\right), \\
& T_{i}=T_{i}^{(0)}+h k T_{i}^{(1)}+\frac{(h k)^{2}}{2} T_{i}^{(2)}+O\left(h^{3} k^{3}\right) .
\end{aligned}
$$

The quantities $R_{i}^{(0)}, R_{i}^{(1)}, R_{i}^{(2)}, T_{i}^{(0)}, T_{i}^{(1)}, T_{i}^{(2)}, S_{i}(0)$, and $U_{i}(0)$ are unknown constants to be determined from the boundary conditions (continuity of the tangential electricand magnetic-field components at the grating boundaries). $\quad R_{i}^{(0)}$ and $T_{i}^{(0)}$ are the reflection and transmission coefficients of the $i$ th space-harmonic field components for a null grating thickness. $R_{i}^{(1)}, R_{i}^{(2)}, T_{i}^{(1)}$, and $T_{i}^{(2)}$ are dimensionless coefficients that represent slight deviation from the asymptotic limit $(h k \ll 1)$. Using the powerseries expansions and identifying terms of the same degree in $h k$, one can easily show that all the unknown constants can be determined. For the zeroth reflection and transmission coefficients $R_{0}$ and $T_{0}$, which are of interest in the following, we find that

$$
\begin{aligned}
R_{0}= & \frac{n_{1}-n_{3}}{n_{1}+n_{3}}\left\{1+j 2 n_{1} \frac{\epsilon_{0}-n_{3}^{2}}{n_{3}^{2}-n_{1}^{2}} h k+\frac{4 n_{1}}{n_{3}^{2}-n_{1}^{2}}\right. \\
& \times\left[j \sum_{p \neq 0} \frac{\epsilon_{p} \epsilon_{-p}}{\left(p^{2} \alpha^{2}-n_{3}^{2}\right)^{1 / 2}+\left(p^{2} \alpha^{2}-n_{1}^{2}\right)^{1 / 2}}\right. \\
& \left.\left.+\frac{\left(n_{1} n_{3}+\epsilon_{0}\right)\left(\epsilon_{0}-n_{3}^{2}\right)}{n_{1}+n_{3}}\right] \frac{(h k)^{2}}{2}\right\}+O\left(h^{3} k^{3}\right), \\
T_{0}= & \frac{2 n_{1}}{n_{1}+n_{3}}\left\{1-j \frac{\epsilon_{0}+n_{1} n_{3}}{n_{1}+n_{3}} h k+\left[\epsilon_{0}-\frac{2 j}{n_{1}+n_{3}}\right.\right. \\
& \times \sum_{p \neq 0} \frac{\epsilon_{p} \epsilon_{-p}}{\left(p^{2} \alpha^{2}-n_{3}^{2}\right)^{1 / 2}+\left(p^{2} \alpha^{2}-n_{1}^{2}\right)^{1 / 2}} \\
& \left.\left.-2 \frac{\left(n_{1} n_{3}+\epsilon_{0}\right)^{2}}{\left(n_{1}+n_{3}\right)^{2}}\right] \frac{(h k)^{2}}{2}\right\}+O\left(h^{3} k^{3}\right) .
\end{aligned}
$$

Let us now consider a situation such that the grating of Fig. 1 is replaced by a homogeneous thin film. We also assume that the relative permittivity $\eta$ of this thin film is dependent on the thin-film thickness and can be written as

$$
\eta=\eta_{0}+h k \eta_{1}+O\left(h^{2} k^{2}\right) .
$$

In Eq. (8) $\eta_{0}$ and $\eta_{1}$ are dimensionless coefficients, and $\eta_{0}$ is the relative permittivity of an infinitely thin film layer. The transmission and reflection coefficients $R_{\text {film }}$ and $T_{\text {film }}$ of the single film, inserted between two semi-infinite media of refractive indices $n_{1}$ and $n_{3}$, can be derived in a closed form. They depend on $n_{1}, n_{3}, \eta, k$, and $h$ and can be expanded in a power series of $h k$. Using Eqs. (57) and (58) of Ref. 17 and Eq. (8) of this paper, we find that

$$
\begin{aligned}
R_{\text {film }}= & \frac{n_{1}-n_{3}}{n_{1}+n_{3}}\left\{1+j 2 n_{1} \frac{\eta_{0}-n_{3}^{2}}{n_{3}^{2}-n_{1}^{2}} h k+\frac{4 n_{1}}{n_{3}^{2}-n_{1}^{2}}\right. \\
& \left.\times\left[j \eta_{1}+\frac{\left(n_{1} n_{3}+\eta_{0}\right)\left(\eta_{0}-n_{3}^{2}\right)}{n_{1}+n_{3}}\right] \frac{(h k)^{2}}{2}\right\} \\
& +O\left(h^{3} k^{3}\right), \\
T_{\text {film }}= & \frac{2 n_{1}}{n_{1}+n_{3}}\left\{1-j \frac{\eta_{0}+n_{1} n_{3}}{n_{1}+n_{3}} h k\right. \\
& \left.+\left[\eta_{0}-\frac{2 j \eta_{1}}{n_{1}+n_{3}}-2 \frac{\left(n_{1} n_{3}+\eta_{0}\right)^{2}}{\left(n_{1}+n_{3}\right)^{2}}\right] \frac{(h k)^{2}}{2}\right\} \\
& +O\left(h^{3} k^{3}\right) .
\end{aligned}
$$

The homogenization is performed by comparing Eqs. (7) and (9). For small grating depths the reflection and transmission coefficients of the grating and of the thin film are identical provided that $\eta_{0}$ and $\eta_{1}$ are equal to $\epsilon_{0}$ and

$$
\sum_{p \neq 0} \frac{\epsilon_{p} \epsilon_{-p}}{\left(p^{2} \alpha^{2}-n_{3}\right)^{1 / 2}+\left(p^{2} \alpha^{2}-n_{1}{ }^{2}\right)^{1 / 2}}
$$

respectively. The result of this substitution is that the effective relative permittivity $\eta_{\mathrm{TE}}$ in the small-gratingdepth limit is given by

$$
\begin{aligned}
\eta_{\mathrm{TE}}= & \epsilon_{0}+\sum_{p \neq 0} \frac{\epsilon_{p} \epsilon_{-p}}{\left(p^{2} \alpha^{2}-{n_{3}}^{2}\right)^{1 / 2}+\left(p^{2} \alpha^{2}-n_{1}{ }^{2}\right)^{1 / 2}} h k \\
& +O\left(h^{2} k^{2}\right)
\end{aligned}
$$

A similar approach can be used to derive the effective properties for TM polarization (magnetic-field vector parallel to the grating vector) of $1-\mathrm{D}$ gratings. The computational methodology, which is similar to that for the TE polarization case, is not given in this paper for the sake of conciseness. The final result for the effective permittivity $\eta_{\mathrm{TM}}$ in the TM polarization case is

$$
\begin{aligned}
\eta_{\mathrm{TM}}= & \epsilon_{0}-\sum_{p \neq 0} \frac{\epsilon_{p} \epsilon_{-p}}{n_{3}{ }^{2}\left(p^{2} \alpha^{2}-{n_{3}}^{2}\right)^{-1 / 2}+n_{1}{ }^{2}\left(p^{2} \alpha^{2}-n_{1}{ }^{2}\right)^{-1 / 2}} \\
& \times h k+O\left(h^{2} k^{2}\right) .
\end{aligned}
$$

Although more handwritten calculus is required, a similar derivation can be done for 2-D gratings. We denote the $y$ component of the grating vector by $K$, the $x$ component of the grating vector by $\rho K$, and the $(m, n)$ th Fourier coefficient of the grating relative permittivity by $\epsilon_{m, n}$. As for the 1-D case, $\alpha$ is equal to $K / k$. For 2-D gratings two polarizations still have to be considered at normal incidence. The incident plane wave is polarized along either the $x$ axis or the $y$ axis. We denote by $\eta_{x}$ and $\eta_{y}$ the corresponding effective relative permittivities. We obtain 


$$
\begin{aligned}
& \eta_{x}=\epsilon_{0,0}-\sum_{(p, q) \neq(0,0)} \frac{\epsilon_{-p,-q} \epsilon_{p, q} C_{p, q}}{A_{p, q}{ }^{2}-C_{p, q} C_{p, q}^{\prime}} h k+O\left(h^{2} k^{2}\right), \\
& \eta_{y}=\epsilon_{0,0}-\sum_{(p, q) \neq(0,0)} \frac{\epsilon_{-p,-q} \epsilon_{p, q} C_{p, q}^{\prime}}{A_{p, q}{ }^{2}-C_{p, q} C_{p, q}^{\prime}} h k+O\left(h^{2} k^{2}\right) .
\end{aligned}
$$

In Eqs. (12) $C_{p, q}, C_{p, q}^{\prime}$, and $A_{p, q}$ are defined by

$$
\begin{aligned}
& C_{p, q}=N_{3 p, q}+N_{1 p, q}-q^{2} \alpha^{2}\left(1 / N_{1 p, q}+1 / N_{3 p, q}\right), \\
& C_{p, q}^{\prime}=N_{3 p, q}+N_{1 p, q}-p^{2} \rho^{2} \alpha^{2}\left(1 / N_{1 p, q}+1 / N_{3 p, q}\right),
\end{aligned}
$$

$$
A_{p, q}=p q \rho \alpha^{2}\left(1 / N_{1 p, q}+1 / N_{3 p, q}\right),
$$

and $N_{i p, q}$ is equal to $\left[\left(p^{2} \rho^{2}+q^{2}\right) \alpha^{2}-n_{i}{ }^{2}\right]^{1 / 2}$, with $i$ $=1,3$. In general, Eqs. (12a) and (12b) provide similar but different expressions for $\eta_{x}$ and $\eta_{y}$. When $\rho$ is equal to 1 and when the grating is symmetric $\left(\epsilon_{m, n}=\epsilon_{-m, n}\right.$ $\left.=\epsilon_{m,-n}=\epsilon_{-m,-n}\right)$, it is easily seen that $C_{p, q}$ and $C_{q, p}^{\prime}$ are equal, and thus $\eta_{x}$ and $\eta_{y}$ are also equal (no polarization effect).

\section{APPROXIMATE EFFECTIVE INDEX DEPENDENCE WITH THE GRATING DEPTH}

Equations (10)-(12) can be simplified by neglecting the $n_{i}{ }^{2}$ dependency in $\left(p^{2} \alpha^{2}-n_{i}{ }^{2}\right)^{1 / 2}$ and in the square-root expressions of $N_{i p, q}$. This approximation amounts to considering that the grating is operating as a zero-order filter with $\alpha$ much larger than the cutoff value, $\alpha_{C}$ $=\max \left(n_{1}, n_{3}\right)$. We obtain the following expressions for TE and TM polarizations of 1-D gratings:

$$
\begin{aligned}
& \tilde{\eta}_{\mathrm{TE}}=\epsilon_{0}+\sum_{p \neq 0} \frac{\epsilon_{p} \epsilon_{-p}}{2|p| \alpha} h k+O\left(h^{2} k^{2}\right), \\
& \tilde{\eta}_{\mathrm{TM}}=\epsilon_{0}-\sum_{p \neq 0} \frac{|p| \alpha \epsilon_{p} \epsilon_{-p}}{n_{3}{ }^{2}+n_{1}{ }^{2}} h k+O\left(h^{2} k^{2}\right) ;
\end{aligned}
$$

and for 2-D gratings we obtain

$$
\begin{aligned}
\tilde{\eta}_{x}= & \epsilon_{0,0}-\left[\sum_{p \neq 0, q \neq 0} \frac{\left(p^{2} \rho^{2}+q^{2}\right)^{3 / 2} \alpha \epsilon_{p, q} \epsilon_{-p,-q}}{2 q^{2}\left({n_{1}}^{2}+n_{3}{ }^{2}\right)}\right. \\
& \left.+\sum_{p \neq 0} \frac{|p| \rho \alpha \epsilon_{p, 0} \epsilon_{-p, 0}}{n_{1}{ }^{2}+n_{3}{ }^{2}}-\sum_{q \neq 0} \frac{\epsilon_{0, q} \epsilon_{0,-q}}{2|q| \alpha}\right] h k \\
& +O\left(h^{2} k^{2}\right), \\
\tilde{\eta}_{y}= & \epsilon_{0,0}-\left[\sum_{p \neq 0, q \neq 0} \frac{\left(p^{2} \rho^{2}+q^{2}\right)^{3 / 2} \alpha \epsilon_{p, q} \epsilon_{-p,-q}}{2 p^{2} \rho^{2}\left(n_{1}{ }^{2}+n_{3}{ }^{2}\right)}\right. \\
& \left.+\sum_{q \neq 0} \frac{|q| \alpha \epsilon_{0, q} \epsilon_{0,-q}}{n_{1}{ }^{2}+n_{3}{ }^{2}}-\sum_{p \neq 0} \frac{\epsilon_{p, 0} \epsilon_{-p, 0}}{2|p| \rho \alpha}\right] h k \\
& +O\left(h^{2} k^{2}\right) .
\end{aligned}
$$

In Eqs. (14) the tilde is used to signify that these effective relative permittivity expressions are valid only for large values of $\alpha$. Equations (14) show that, for infinitely small depths, the birefringence properties of gratings vanish; $\epsilon_{0}$ and $\epsilon_{0,0}$ are the common effective relative permittivities of 1-D and 2-D gratings, respectively, for any polarization direction of the normally incident plane wave. However, we note that this result holds only in the asymptotic limit and that, for nonzero depth (even if it is very small), the gratings exhibit birefringence properties.

As shown by Eqs. (10)-(12), the effective relative permittivities in the small-depth limit depend not only on the grating parameters but also on the optical indices of the surrounding media; the grating effective properties are not intrinsic to the periodic structure but are rather related to the whole diffraction problem. However, as can be shown by Eq. (14a), for large values of $\alpha$ and for TE polarization of $1-\mathrm{D}$ gratings, the effective relative permittivity depends only on the grating parameters. In that case the effective properties are intrinsic to the periodic structures. This is not true for the TM polarization case. According to Eq. (14b), $\tilde{\eta}_{\mathrm{TM}}$ depends on the optical indices $n_{1}$ and $n_{3}$. Since this was unexpected, we decided to confirm this prediction with rigorous computations. So let us consider a sinusoidally modulated grating with a relative permittivity $\epsilon_{r}$ given by

$$
\epsilon_{r}=\epsilon_{0}+\Delta \epsilon \cos (K x) .
$$

Throughout the paper and for numerical purposes $\epsilon_{0}$ and $\Delta \epsilon$ are equal to 5 and 3 , respectively. The high index modulation is chosen because it allows a better testing of EMT predictions that are, in general, less accurate in situations strongly departing from the homogeneous case.

Figure 2 shows the comparison between rigorous computation results and the prediction of Eq. (14b). For the computation RCWA (Ref. 18) was used. In this special TM polarization case the formulation with a highly improved convergence rate ${ }^{19}$ was implemented. In Fig. 2 the solid curve is obtained with Eq. (14b) for substrate optical indices varying between 2.6 and 3.5. Crosses represent effective relative permittivities derived from rigorous computations, as explained in Section 4. Good agree-

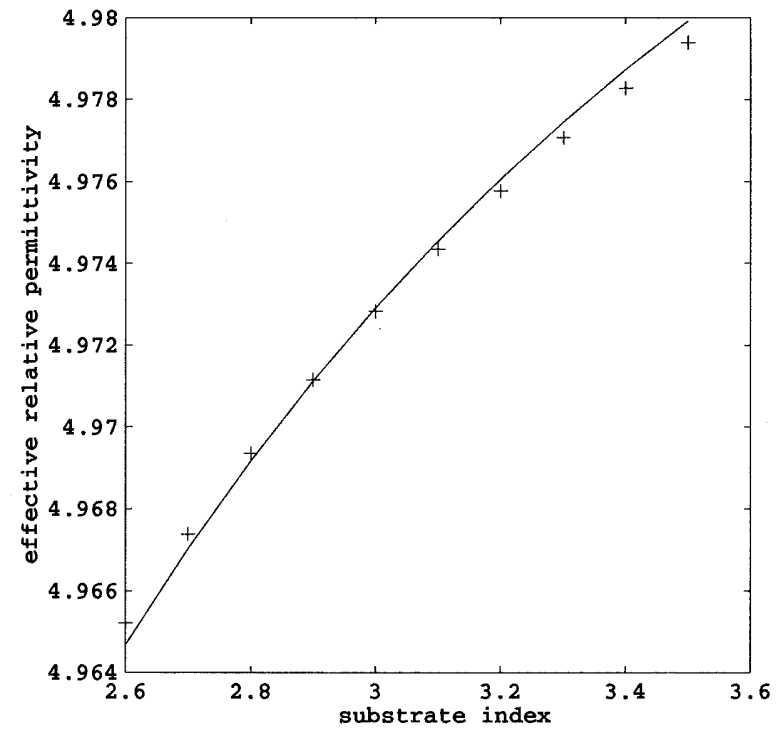

Fig. 2. Dependence of the effective relative permittivity with the substrate optical index. Crosses are obtained with rigorous computations, and the solid curve corresponds to the $\tilde{\eta}_{\mathrm{TM}}$ prediction of Eq. (14b). $h=0.001 \lambda, \alpha=10$, and $n_{1}=1$. 
ment between the prediction and rigorous computations is found. This confirms the nonintrinsic behavior of the effective properties of 1-D gratings for TM polarization.

In Section 2 the effective properties of gratings are derived in the limit of small depth-to-wavelength ratios, which means that $h / \lambda \rightarrow 0$ while $\Lambda / \lambda$ is held constant. This approach strongly differs from that of classical EMT results (see Refs. 10-12), where the depth-to-wavelength ratio is held constant while $\Lambda / \lambda \rightarrow 0$. Note also that, in a previous theoretical work, ${ }^{20}$ Wirgin considered a grating with fixed groove depth and period and allowed the wavelength of the incident wave to become increasingly large. Consistently with Eqs. (14a) and (14b), he found that the effective relative permittivity of 1-D gratings is $\epsilon_{0}$ for any polarization of the incident light. All these results obtained with different assumptions are a little confusing. To avoid any confusion in the following, we will refer to the EMT prediction of Eqs. (10)-(12) as the smalldepth limit and to the classical EMT results with $\Lambda / \lambda \rightarrow 0$ and $h / \lambda$ held constant as the large-depth limit. The work by Wirgin will not be considered. In the following we denote by $n_{L}$ the effective index in the large-depth limit. This effective index, which does not depend on the surrounding region, is intrinsic to the periodic structure. When deriving the expressions for $n_{L}$, no boundary conditions are involved and the periodic structure is assumed to have an infinite spatial extent $(h \rightarrow \infty)$. This is obvious from the theoretical derivations in Refs. 10 and 12 and is particularly enlightened in the paper by Bouchitté and Petit, ${ }^{21}$ where a rigorous mathematical formalism is applied in homogenization theory. In general, the effective index $n_{L}$ is expanded in a power series of $\alpha^{-1}$, and the series coefficients depend on the Fourier coefficients of the grating relative permittivity. ${ }^{11,12}$

From the knowledge of grating effective properties in the small-and large-depth limits an approximate expression of the effective index $n(h)$ for arbitrary grating depths is derived. We assume that the effective index $n(h)$ can be set in the following form:

$$
n^{2}(h)=\eta_{0}+\frac{2}{\pi}\left(n_{L}^{2}-\eta_{0}\right) \arctan \left(\pi^{2} \frac{\eta_{1}}{n_{L}{ }^{2}-\eta_{0}} \frac{h}{\lambda}\right) .
$$

In Eq. (16) the arctangent dependency is somewhat arbitrary, but it fulfills the three conditions $n^{2}(0)=\eta_{0}$, $\mathrm{d} n^{2} / \mathrm{d}(h k)=\eta_{1}$, and $n^{2}(\infty)=n_{L}{ }^{2}$. Equation (16) will be used in Section 4 for comparison with rigorous computation results.

\section{SIMULATION RESULTS}

In order to verify the depth dependence prediction, we proceed as follows. Figure 1 defines a diffraction problem that is solved by RCWA assuming a normal incident plane wave of unit amplitude. The grating depth $h$ is varied between $0.01 \lambda$ and either $1.5 \lambda$ or $2 \lambda$ according to the specific case investigated in the computation. By solving Maxwell's equations, we compute the complex transmitted amplitude $t$ and reflected amplitude $r$ of the zero-order diffracted waves. Note that in all our simulations the period-to-wavelength ratio never exceeds the cutoff value beyond which non-zero-order waves propa-

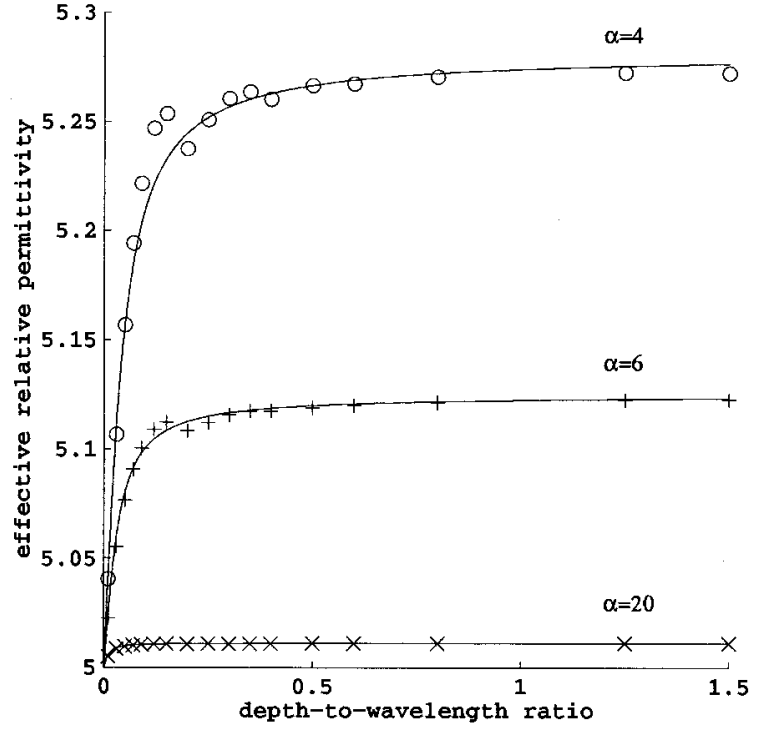

Fig. 3. TE polarization of a sinusoidally modulated grating $\left(\epsilon_{0}\right.$ $=5, \Delta \epsilon=3, n_{1}=1$, and $n_{3}=3$ ). Crosses, plus signs, and circles are rigorous computation results obtained for $\alpha=20,6$, and 4, respectively. Solid curves are obtained from Eq. (16) with $n_{L}$ given by Eq. (18a).

gate in either the substrate or the incident medium. Then, to make the analogy between subwavelength periodic structures and homogeneous media, we define the equivalent effective index of the periodic structure by the real number $n_{\mathrm{RCWA}}$, which minimizes the error function

$$
e=\left|r^{\prime}\left(n_{\mathrm{RCWA}}\right)-r\right|^{2}+\left|t^{\prime}\left(n_{\mathrm{RCWA}}\right)-t\right|^{2},
$$

where $r^{\prime}\left(n_{\mathrm{RCWA}}\right)$ and $t^{\prime}\left(n_{\mathrm{RCWA}}\right)$ are the reflection and transmission complex coefficients of a thin homogeneous layer of thickness $h$ and optical index $n_{\text {RCWA }}$, inserted between two semi-infinite media of optical indices $n_{1}$ and $n_{3}$. Since the effective index $n_{\text {RCWA }}$ is expected to be larger than the minimal value $n_{\text {min }}$ of the grating index and smaller than the maximal value $n_{\max }$ of the grating index, the minimization of the error function is restricted to the interval $\left[n_{\min }, n_{\text {max }}\right]$. A similar approach was used in Ref. 12 to verify the validity of the large-depthlimit EMT prediction.

In the two following tests a 1-D grating with the sinusoidal index modulation of Eq. (15) is considered. According to Eqs. (8) and (15) of Ref. 12, the effective index $n_{L}$ in the large-depth limit is

$$
\begin{array}{ll}
\text { TE: } & n_{L}^{2}=\epsilon_{0}+\frac{1}{2} \Delta \epsilon^{2} \alpha^{-2}+O\left(\alpha^{-4}\right), \\
\text { TM: } & n_{L}^{2}=\frac{1}{a_{0}}+\frac{a_{1}}{a_{0}{ }^{3}}\left(\frac{\Delta \epsilon a_{2}}{2}+2 \epsilon_{0} a_{1}\right) \alpha^{-2}+O\left(\alpha^{-4}\right) .
\end{array}
$$

In Eq. (18b) the $a_{i}$ 's are the Fourier coefficients of the inverse relative permittivity. The first simulation result shown in Fig. 3 is obtained for TE polarization. Three values of $\alpha$ are considered. $\alpha=20,6$, and 4. Crosses, plus signs, and circles correspond to rigorous computation results obtained by minimizing the error function of Eq. (17). In Fig. 3 the solid curves correspond to the effective index prediction of Eq. (16) with $n_{L}$ given by Eq. (18a). 
These curves are in good agreement with the rigorous results, especially for $\alpha=20$. For $\alpha=6$ and 4 a slight deviation is observed for $h / \lambda$ values of approximately 0.2 . Over the entire depth interval considered, the discrepancy $\Delta n$ between the effective indices obtained with rigorous computation and with EMT never exceeds $1 \%$ $(\Delta n / n<0.003)$.

The second result, shown in Fig. 4, concerns the TM polarization case. As above, the same values of $\alpha$ and of the index modulation are considered. For the large-depth limit the effective index of Eq. (18b) is considered. Three regions can be distinguished in Fig. 4. For large depthto-wavelength ratios $(h / \lambda>0.4)$ we observe a good agreement between the EMT prediction and rigorous computations. The maximum deviation $\Delta n$ is smaller than 0.05 for $\alpha=4,0.03$ for $\alpha=6$, and 0.01 for $\alpha=20$. For small depth-to-wavelength ratios a good agreement is obtained, and the effective properties are well described by Eq. (14b), for any value of $\alpha$.

Of more interest is the third region, defined by $h / \lambda$ values larger than 0.1 and smaller than 0.4. The arctangent formula of Eq. (16) deviates from the rigorous results. The largest deviation $(\Delta n=0.06)$ is observed for $\alpha=4$ and 6 and for $h / \lambda=0.15$. It corresponds to a relative error $(\Delta n / n)$ of $3 \%$. In this region rigorous computations show that the effective indices are much smaller than the large-depth-limit prediction. We could not explain this anomalous behavior, but when we computed $n_{\text {RCWA }}$ by minimizing the error function of Eq. (17), we noted that the largest errors were observed in this region (although the error $e$ was always found to be smaller than 0.05). The third result concerns a 2 -D grating with a relative permittivity $\epsilon_{r}$ equal to

$$
\epsilon_{r}=\epsilon_{0}+\frac{\Delta \epsilon}{2} \cos (K x)+\frac{\Delta \epsilon}{2} \cos (K y) .
$$

As in Section 3, $\epsilon_{0}$ and $\Delta \epsilon$ are equal to 5 and 3, respectively. For this symmetric structure no polarization effects occur. An incident plane wave polarized along the $x$

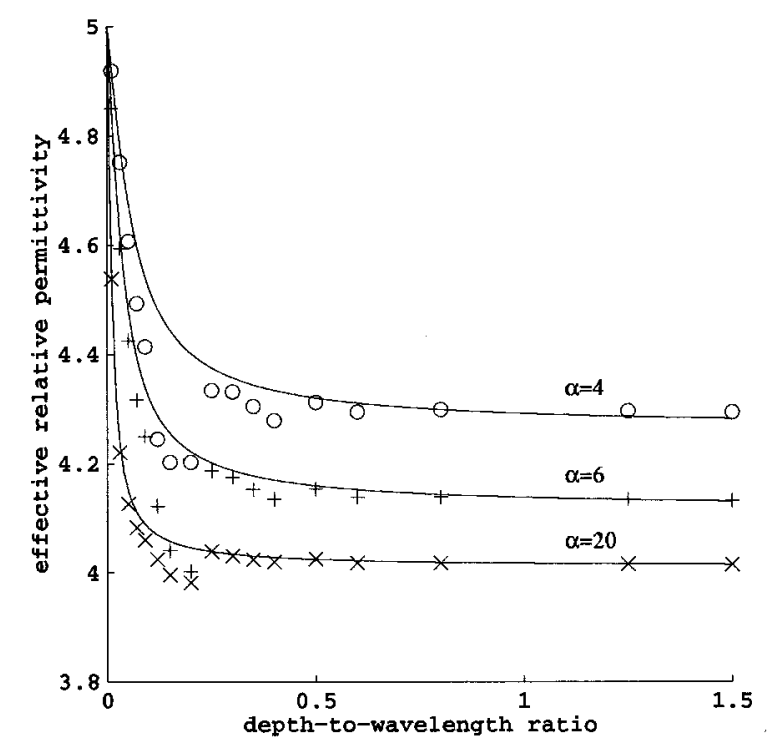

Fig. 4. Same as Fig. 3 (for TM polarization), except that the effective index $n_{L}$ in the large-depth limit is given by Eq. (18).

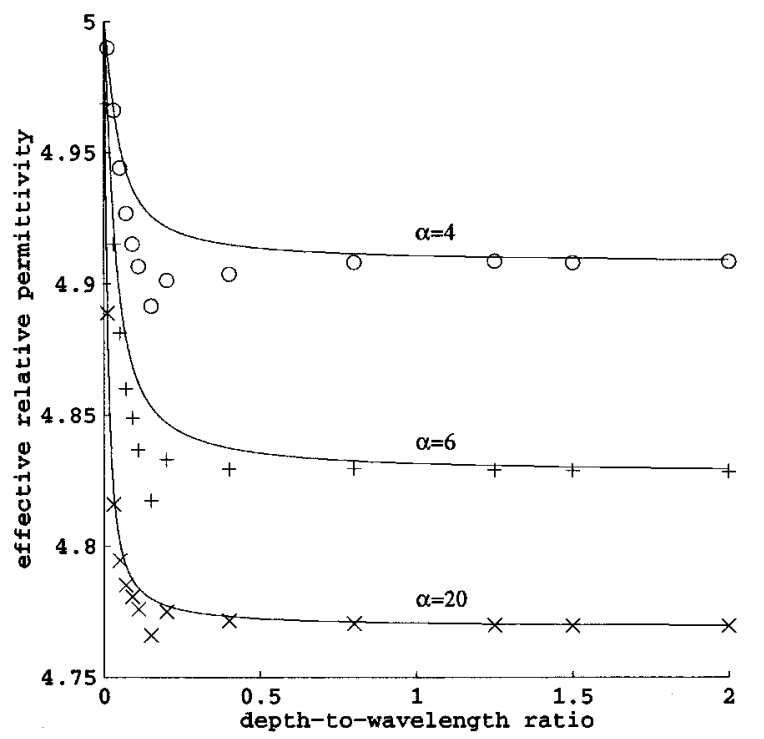

Fig. 5. Comparison between the EMT prediction of Eq. (16) and rigorous computation results for the 2-D grating whose relative permittivity is given by Eq. (19). Crosses, plus signs, and circles are defined as for Fig. 3.

direction is considered for the computation. In the largedepth limit no closed-form expressions for the effective index $n_{L}$ exist to our knowledge. As shown in Ref. 12, the computation of the zero- and second-order terms of the power-series expansion requires the inversion of a matrix A that must first be truncated for numerical purposes. In the case of a sinusoidally modulated periodic structure, convergence problems that are due to the truncation of matrix $\mathbf{A}$ are not encountered. This is due to the existence of only five nonzero coefficients in the Fourier expansion of the grating relative permittivity. This is also especially true for the rigorous computation; 81 orders retained in our code ${ }^{22}$ were enough to ensure the convergence (the maximum difference between the transmitted and reflected zero-order intensities computed with 49 and 81 retained orders does not exceed $10^{-2} \%$ in our computations).

A comparison between the EMT prediction of Eq. (16) and the rigorous computation results is shown in Fig. 5. The results are slightly better than those of the TM polarization case, and again three regions may be distinguished. This is not surprising if one considers that 2-D gratings can be approximated as a superposition ${ }^{15}$ of two 1-D gratings illuminated with TE and TM polarizations. For the 2-D case the discrepancy observed in the intermediate region is smaller than that of the TM polarization case. More precisely, for any values of $\alpha$ and of $h / \lambda$, the effective index discrepancy $\Delta n$ between the arctangent expression and rigorous computation results never exceeds 0.04 (relative error $\Delta n / n$ less than $0.8 \%$ ). This implies that the arctangent expression can be used with relative confidence for predicting the effective properties of 2-D volume gratings. It is interesting to note that, for this particular case in which no convergence problems occur, an excellent agreement between the EMT prediction and rigorous results is obtained in the largedepth limit. The difference is almost imperceptible in Fig. 5, even for values of $\alpha$ just smaller than the cutoff 
value $\alpha_{C}=3$. This is a strong confirmation of the validity of the EMT derivation of Ref. 12.

When one is considering gratings with a noncontinuous relative permittivity (for instance, lamellar gratings), the power-series expansion used in Section 2 is no longer valid for TM polarization of 1-D gratings and for 2-D gratings. This is evident from Eqs. (11) and (12), since the $\eta_{1}$ coefficients are infinite. In Eq. (11) the summation over $p$ becomes increasingly large as the logarithm of $p$. This does not imply that the effective index of lamellar gratings is not defined but simply means that the methodology of Section 2 cannot be applied. However, from Eqs. (11) and (12) and according to the classical results on the EMT in the large-depth limit, it is predicted that the effective indices of 1-D lamellar gratings for TM polarization and of 2-D lamellar gratings are respectively $\epsilon_{0}$ and $\epsilon_{0,0}$ for a null grating thickness. It is also predicted that the effective indices present a strong and sharp variation at very small thicknesses (vertical slope at $h=0$ ).

In Fig. 6 rigorous computation results obtained by minimizing the error function of Eq. (17) are shown for 1-D and 2-D lamellar gratings. The 1-D lamellar grating considered has one groove per period, and the fill factor defined by the groove width-to-period ratio is equal to 0.5 . The 2-D grating is composed of square features. The fill factor in each direction is equal to 0.5 , and the periods along each direction are equal. The gratings are etched in a substrate with an optical index $n_{3}$ equal to 3 , and the incident medium is air $\left(n_{1}=1\right)$, so the high and low optical indices of the gratings are equal to 3 and 1, respectively. Crosses, plus signs, and circles correspond to rigorous computation results obtained for $\alpha=20,6$, and 4 , respectively. For the rigorous computation of the reflected and transmitted amplitudes of the 1-D grating, 301 orders were retained in the computation. For the 2-D grating, 289 orders were retained. This corresponds to \pm 8 orders in each direction and is the maximum number of orders that can be retained with our workstation. As was noted in Table 2 of Ref. 12, convergence is not guaranteed, and only approximate values of $n_{\mathrm{RCWA}}$ are computed.

In Fig. 6(a) the solid curves correspond to the effective index prediction of Eq. (16) for TE polarization. The summation over $p$ in Eq. (14a) was truncated to \pm 120 terms for numerical purposes. This was largely sufficient to ensure a five-digit accuracy for the computation of $\eta_{1}$. As in the sinusoidally modulated grating case, good agreement between EMT and the rigorous computation is obtained. For TM polarization [Fig. 6(b)] and for the 2-D grating [Fig. 6(c)] the effective relative permittivities in the large-depth limit are plotted as horizontal lines, since the arctangent formula is not valid. For the computation of the zero and second orders of the effective index $n_{L}$ of the 2-D grating the same number of Rayleigh orders used in the rigorous computation are retained for the matrix inversion ( $M_{\mathrm{EMT}}=8$ in Ref. 12). Basically, the qualitative predictions obtained from Eqs. (11) and (12) are verified, and the same conclusions as those for the volumegrating case hold. For small depth-to-period ratios the effective relative permittivities drop quickly and tend to be constant in the large-depth limit. However, we note that, for large depths, the effective relative permittivity

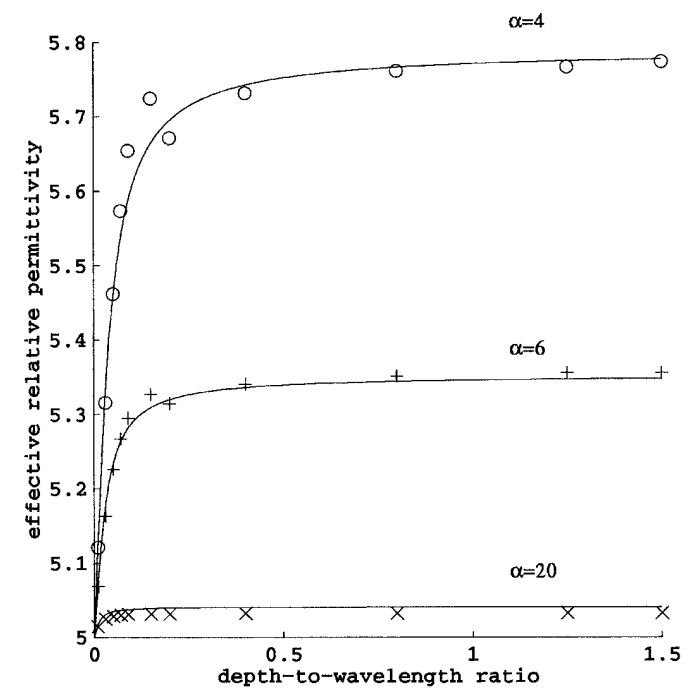

(a)

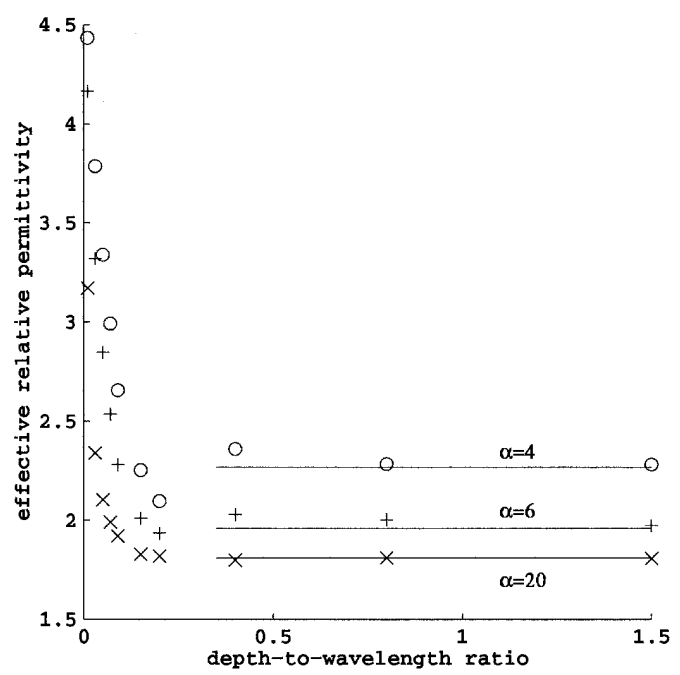

(b)

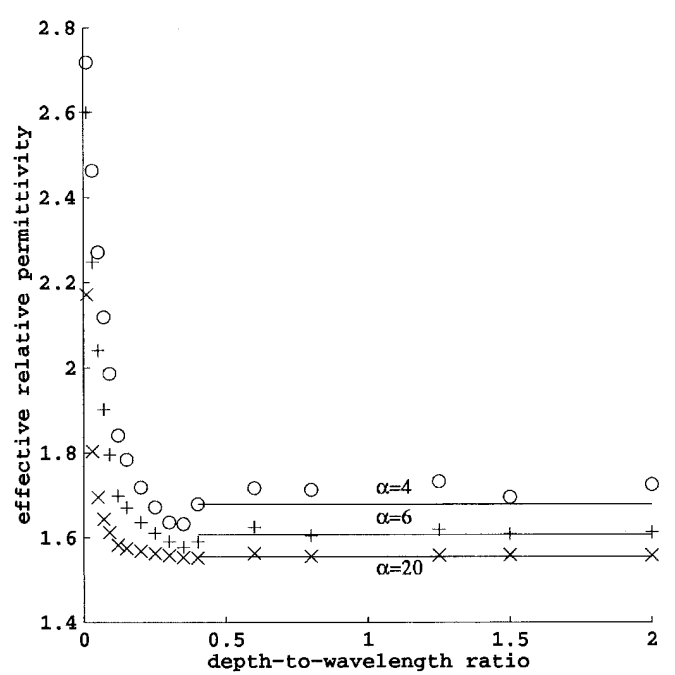

(c)

Fig. 6. EMT of lamellar gratings: (a) TE polarization of a 1-D grating, (b) TM polarization of a 1-D grating, and (c) 2-D grating. In (a) the solid curve corresponds to Eq. (16), and in (b) and (c) the solid lines correspond to the EMT prediction of Ref. 12 in the large-depth limit. 
slightly fluctuates in Fig. 6(c), especially for $\alpha=4$. These fluctuations are rather small; for the six larger values of $h / \lambda$ the mean value and the standard deviation of the effective index $n_{\text {RCWA }}$ are equal to 1.320 and 0.006 , respectively. We cannot explain the origin of these fluctuations, which were not previously observed in Fig. 5 , but convergence problems encountered with the rigorous computation may be responsible.

\section{DISCUSSION AND CONCLUSION}

In this paper we considered one-dimensional (1-D) and two-dimensional (2-D) gratings with infinitely small thicknesses. By solving Maxwell's equations in the grating region and by matching the boundary conditions, we expanded the reflected and transmitted zero-order diffracted amplitudes in a power series of the grating thickness. Closed-form expressions for grating effective indices were derived by comparing these amplitudes with those of a homogeneous thin film with the same thickness. It was shown that the effective properties in the small-depth limit are not intrinsic to the grating but depend on the whole diffraction problem and especially on the optical indices of the homogeneous media surrounding the grating region. Much simpler expressions for the effective indices were derived for periods small compared with the wavelength. Within this approximation and for TE polarization the effective indices of 1-D gratings stop depending on the optical indices of the surrounding media and become intrinsic to the grating structures. This is not true for TM polarization of 1-D gratings and for 2-D gratings.

The above results derived in the small-depth limit were combined with classical results from the effective-medium theory (EMT) of periodic structures. Arctangent expressions for the effective indices of 1-D and 2-D gratings were derived for arbitrary grating thicknesses. These expressions are valid for volume gratings and for TE polarization of 1-D surface-relief gratings. For TM polarization of 1-D surface-relief gratings and for 2-D surfacerelief gratings, only a qualitative description of the depth effective index dependence was obtained. Comparison of the arctangent expressions with rigorous computation results shows good agreement for TE polarization of 1-D gratings. For TM polarization and for 2 -D volume gratings we noticed a discrepancy. This discrepancy is observed for intermediate grating thicknesses of approximately 0.2 wavelength. Although it is weak, especially for 2-D volume gratings, the discrepancy cannot be ignored and it can be recommended that one use rigorous computation to assess the effective properties accurately.

From a practical point of view much care must be taken when using EMT for designing antireflection subwavelength gratings. According to Fig. 6, for quarter-wave grating depths, the EMT in the large-depth limit, which is usually considered for the design, is not valid. Even if the arctangent formula can be used with confidence for TE polarization of 1-D gratings, for TM polarization, and for 2-D gratings, rigorous computation is preferable for optimizing grating structures. When larger grating depths are considered, for instance for graded-phase diffractive elements, the classical EMT prediction is accu- rate. Our experience shows that quasi-optimal designs can be obtained with EMT and that refinements with rigorous computations are not required. We expect that this work helps clarify some aspects of the effective properties of subwavelength gratings and that the new formulas derived in this paper will be useful for accurately designing subwavelength structures through the EMT.

\section{ACKNOWLEDGMENTS}

When this work began, Philippe Lalanne was a visiting scientist at The Institute of Optics of the University of Rochester. He is pleased to acknowledge the Direction Générale de l'Armement for financial support under contract DRET-DGA 94-1123. The authors are grateful to G. M. Morris, S. Norton, and S. Peng for helpful discussions.

\section{REFERENCES}

1. P. Yeh, "A new optical model for wire grid polarizers," Opt. Commun. 26, 289-292 (1978).

2. S. J. Wilson and M. C. Hutley, "The optical properties of 'moth eye' antireflection surfaces," Opt. Acta 29, 993-1009 (1982).

3. R. C. Enger and S. K. Case, "Optical elements with ultrahigh spatial-frequency surface corrugations," Appl. Opt. 22, 3220-3228 (1983).

4. M. E. Motamedi, W. H. Southwell, and W. J. Gunning, "Antireflection surfaces in silicon using binary optics technology," Appl. Opt. 31, 4371-4376 (1992).

5. D. H Raguin and G. M. Morris, "Antireflection structured surfaces for the infrared spectral region," Appl. Opt. 32, 1154-1167 (1993).

6. S. S. Wang, R. Magnusson, J. S. Bagby, and M. G. Moharam, "Guided-mode resonances in planar dielectriclayer diffraction gratings," J. Opt. Soc. Am. A 8, 1470-1475 (1990).

7. W. Stork, N. Sreibl, H. Haidner, and P. Kipfer, "Artificial distributed-index media fabricated by zero-order gratings," Opt. Lett. 16, 1921-1923 (1991).

8. W. M. Farn, "Binary gratings with increased efficiency," Appl. Opt. 31, 4453-4458 (1992).

9. F. T. Chen and H. G. Craighhead, "Diffractive phase elements on two-dimensional artificial dielectrics," Opt. Lett. 20, 121-123 (1995).

10. S. M. Rytov, "Electromagnetic properties of a finely stratified medium," Sov. Phys. JETP 2, 466-475 (1956).

11. J. M. Bell, G. H. Derrick, and R. C. McPhedran, "Diffraction gratings in the quasi-static limit," Opt. Acta 29, 1475-1489 (1982).

12. Ph. Lalanne and D. Lemercier-Lalanne, "On the effective medium theory of subwavelength periodic structures," J. Mod. Opt. 43, 2063-2085 (1996).

13. J. L. Jackson, and S. R. Coriell, "Transport coefficients of composite materials," J. Appl. Phys. 39, 2349-2354 (1968).

14. E. B. Grann, M. G. Moharam, and D. A. Pommet, "Artificial uniaxial and biaxial dielectrics with use of two-dimensional subwavelength binary grating," J. Opt. Soc. Am. A 11, 2695-2703 (1994)

15. R. Bräuer and A. Bryngdahl, "Design of antireflection gratings with approximate and rigorous methods," Appl. Opt. 33, 7875-7882 (1994).

16. M. G. Moharam, E. B. Grann, D. A. Pommet, and T. K. Gaylord, "Formulation for stable and efficient implementation of the rigorous coupled-wave analysis of binary gratings," J. Opt. Soc. Am. A 12, 1068-1086 (1995).

17. M. Born and E. Wolf, Principle of Optics, 6th ed. (Pergamon, New York, 1984), p. 62. 
18. M. G. Moharam, E. B. Grann, D. A. Pommet, and T. K. Gaylord, "Formulation for stable and efficient implementation of the rigorous coupled-wave analysis of binary gratings," J. Opt. Soc. Am. A 12, 1068-1076 (1995).

19. Ph. Lalanne and G. M. Morris, "Highly improved convergence of the coupled-wave method for TM polarization," J. Opt. Soc. Am. A 13, 779-784 (1996).

20. A. Wirgin, "On the reduction of reflection between two transparent media by interface roughening," Opt. Commun. 37, 321-325 (1981).

21. G. Bouchitté and R. Petit, "Homogenization techniques as applied in the electromagnetic theory of gratings," Electromagnetics 5, 17-36 (1985).

22. S. Peng and G. M. Morris, "An efficient implementation of rigorous coupled-wave analysis for surface relief gratings," J. Opt. Soc. Am. A 12, 1087-1096 (1995). 